\title{
Effizienzsteigerung und Kostenreduktion durch Straffung der Wundversorgung von chronischen Wunden in einem Klinikverbund mit 16 Kliniken
}

\author{
Increasing Efficiency and Reducing Costs by Streamlining Wound \\ Care for Chronic Wounds in a Hospital Group of 16 Clinics
}

Autoren
Guido Bruning, Enno Marquet, Jasmin Woitalla-Bruning
Institut
Krankenhaus Tabea GmbH \& Co. KG Kösterbergstraße 32
22587 Hamburg
Schlüsselwörter
Wundversorgung, Rationalisierung, Kostenreduktion, Evidenz

Key words

wound care, streamlining, cost reduction, evidence

online publiziert 20.07.2021

Bibliografie

Phlebologie 2021; 50: 283-287

DOI $10.1055 / a-1531-9188$

ISSN 0939-978X

(c) 2021. Thieme. All rights reserved.

Georg Thieme Verlag KG, Rüdigerstraße 14,

70469 Stuttgart, Germany

Korrespondenzadresse

Dr. med. Guido Bruning

Krankenhaus Tabea GmbH \& Co. KG

Zentrum für Venen- und Dermatochirurgie,

Kösterbergstraße 32, 22587 Hamburg, Deutschland

gbruning@tabea-krankenhaus.de

\section{ZUSAMMENFASSUNG}

Seit Jahren gilt die feuchte Wundbehandlung als Standard in der Wundversorgung. Obwohl dieser Grundsatz allgemein akzeptiert ist, ist die Evidenz schon für dieses Dogma überschaubar. Die Industrie bietet auf diesem Grundsatz eine Vielzahl von Produkten zur Wundversorgung an, deren Überle- genheit gegenüber anderen Produkten auf evidenzbasierter Grundlage oft nicht gegeben ist.

Ziel war es, in einer Klinikgruppe das Sortiment unter hauptsächlich einkaufspolitischen Kriterien zu straffen und zu häufige, unbegründete Therapiewechsel zu vermeiden. Daraus ergab sich die Aufgabe, sich nach Möglichkeit auf wenige Hersteller zu begrenzen.

Um die Akzeptanz und die Gründe für alle Anwender im Prozess transparent zu gestalten, wurden diese über eine strukturierte Abfrage für das Thema sensibilisiert und mitgenommen. Als Ergebnis ist ein kleines, sehr straffes Kontingent in der Wundversorgung mit hoher Anwenderakzeptanz entstanden. Hierdurch lassen sich im Einkauf deutliche Kosteneinsparungen realisieren.

\section{ABSTRACT}

For years, moist wound treatment has been considered the standard procedure in wound care. Although this principle is generally accepted, the evidence for this dogma alone is scarce. The industry offers a large variety of wound care products based on this principle, without evidence-based superiority over other products.

The aim was to streamline the product range in a hospital group, in order to reduce unnecessary costs and avoid unjustified changes in therapy. This led to a limited number of only a few suppliers, whenever possible.

All users were involved in the process and asked to fill out a structured questionnaire to raise awareness for this issue and to generate transparency. The result was a small, selective contingent of wound care products, highly accepted by the users, allowing significant cost savings as a consequence.

\section{Einleitung}

In der Wundversorgung von chronischen Wunden, aber auch von akuten Wunden, steht die stadiengerechte Therapie im Vordergrund. Die Industrie bietet hierzu eine Vielzahl von Produkten zur Wundabdeckung und Förderung der Wundheilung an, die alle dem Grundsatz der feuchten Wundversorgung als Überlegenheit zur trockenen Wundversorgung folgen. Allerdings ist auch für die- sen Grundsatz in der Wundversorgung trotz allgemeiner Akzeptanz die Evidenz überschaubar ( $\triangleright$ Abb. 1).

Die Leitlinie der Deutschen Gesellschaft für Wundheilung und Wundbehandlung e. V. der AWMF aus dem Jahr 2012 [1] spiegelt hier das Dilemma in der täglichen Praxis eindeutig wider. Das Prinzip der feuchten Wundbehandlung kombiniert mit der Forderung nach stadiengerechter Wundtherapie führt in der Leitlinie zu einer 

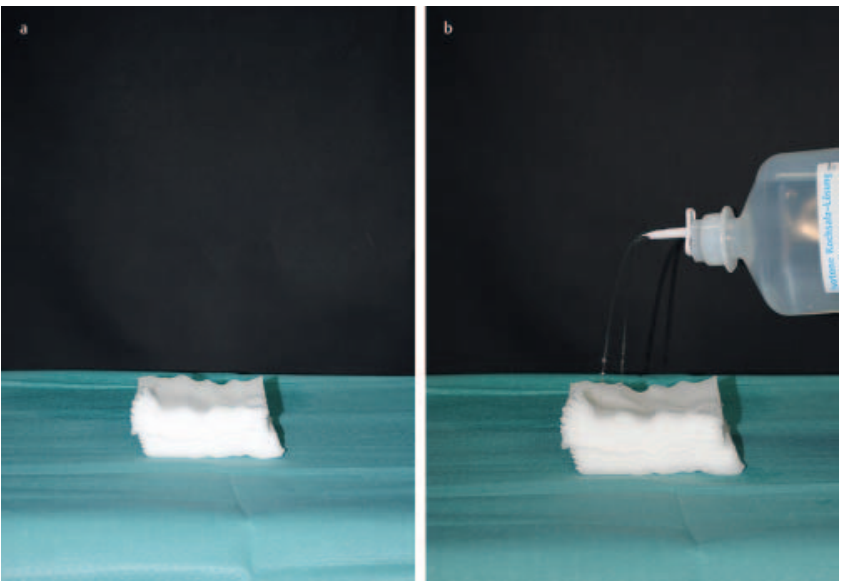

- Abb.1 Grundlage der modernen Wundtherapie ist der Vorzug der feuchten Wundbehandlung. Jedoch ist auch für dieses Dogma die Evidenz überschaubar.

Empfehlung, die auf einer sehr geringen Evidenz basiert. Grund hierfür ist, dass zu den unterschiedlichen Produkten der Industrie kaum vergleichende Daten in guter Qualität zur Verfügung stehen. Nur für einige wenige Produkte liegen Studien vor, die deren Wirksamkeit und Überlegenheit in der Frage der Wundheilung, jedoch oft nur im Vergleich zu einer feuchten Kompresse, belegen.

In der Praxis führt dieses Phänomen dazu, dass insbesondere in den Kliniken ein unübersichtliches Sortiment diversester Wundauflagen verschiedenster Hersteller zur Verfügung steht. Die Auswahl unterliegt den persönlichen Interessen des Behandlers, aber zum Teil auch des Patienten, die durch unterschiedlichste Einflüsse entstanden sind ( $>$ Abb. 2). Vielen ist hierbei nicht bewusst, dass einzelne Klassen der Wundauflagen in der Indikationsstellung identisch sind und die Evidenz in der Überlegenheit verschiedener Wundauflagen fehlt. Dies führt zu einem inkonsistenten, ineffektiven und in verschiedenen Abteilungen unterschiedlichen Versorgungskonzept, das einen erhöhten Ressourcenverbrauch der zum Teil doch sehr teuren Produkte bewirkt. Außerdem führt dies zu häufigen, unbegründeten Therapiewechseln ohne rationale Grundlage.

\section{Klassifikation und Indikationen der Produkt- gruppen in der Wundversorgung}

\section{Wundgaze}

Die Wundgaze soll das Verkleben des Wundsekrets und der Wunde mit der Wundauflage verhindern und so zu schmerzfreieren und atraumatischen Verbandswechseln führen. Sie sind im Krankenhaus ubiquitär verbreitet. Es handelt sich hier um Zellulose oder Kunstfaser, in der Regel mit Salbenimprägnierung, zum Teil unter Zusatz von antiseptischen Wirkstoffen oder lokalen Antibiotika. Bezüglich der Zusatzstoffe bleibt festzustellen, dass die Wirksamkeit von Silberimprägnierungen nicht wirklich nachgewiesen ist sowie der Einsatz von lokalen Antibiotika aufgrund der Resistenzentwicklung massiv eingeschränkt werden sollte. Der Einsatz von Mineralölpro-
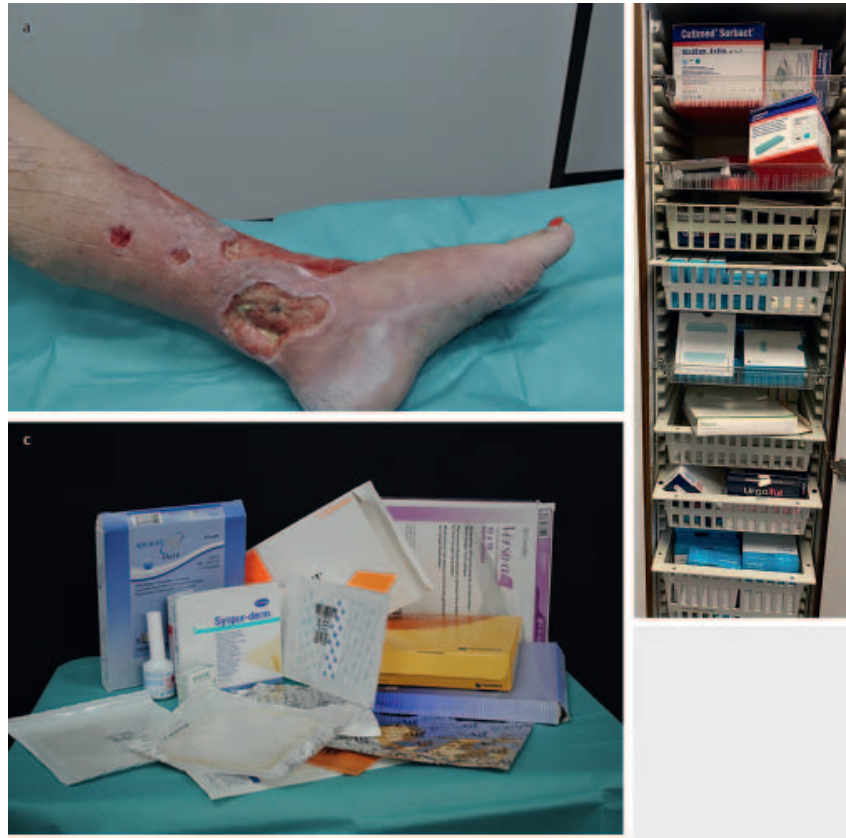

- Abb. 2 Das Dilemma der Wundbehandlung in der täglichen Praxis: Einem Problem steht eine unüberschaubare Zahl von verschiedenen Produkten als Lösung gegenüber.

dukten in größeren Mengen, wie beispielsweise Vaseline, in diesen Wundgazen ist nicht wundheilungsfördernd. Eine Ausnahmestellung nimmt ein Produkt ein, welches durch die Adhäsion von Mikroorganismen und Keimen an der Wundgaze mittels Dialkylcarbamoylchlorid-Bedampfung zu einer Wundreinigung und nachgewiesenen Reduzierung der Keimbesiedelung in der chronischen Wunde allein durch seine physikalischen Eigenschaften führt [2].

\section{Alginate}

Bei Alginaten handelt es sich um ein zelluloseähnliches Polysaccharid aus Seealgen als Salz oder Ester. Sie bieten ein hervorragendes Exsudat-Management bei stark sezernierenden Wunden und können auch in tieferen Wundhöhlen eingesetzt werden. Auch diese Präparate sind in Kombination mit antiseptischen Imprägnierungen wie Silber oder Antiseptika erhältlich [3]. Primär befeuchtet können Alginate auch zur Befeuchtung einer zu trockenen Wunde eingesetzt werden [4]. 


\section{Hydrofaser}

Hierbei handelt es sich um Natrium-Carboxymethylcellulose. Die Indikationsstellung entspricht denen der Alginate. Auch diese sind häufig mit Silber oder Antiseptika imprägniert erhältlich. Sie bieten gegenüber den Alginaten den Vorteil, dass sie länger strukturstabil bleiben und sich leichter aus der Wundhöhle bzw. Wunde wieder entfernen lassen, insbesondere wenn es sich um übernähte Produkte handelt [5].

\section{Hydrogele}

Hydrogele sind als gelförmige Applikationsform in Form von Tuben, aber ebenso in Form von Wundauflagenplatten verfügbar. Sie dienen der Befeuchtung der Wunde, dem Ablösen von Nekrosen und der Verbesserung der Wundbefeuchtung bei zu trockenen Wunden [6].

\section{Hydrokolloide}

Hydrokolloide sind die Pioniere der feuchten Wundbehandlung. Es handelt sich hierbei um Natrium-Carboxymethylcellulosepektin oder-gelatine, welches auf einem Schaumstoff oder auf einem Polyurethanfilm aufgebracht ist. Diese Auflagen können mehrere Tage auf der Wunde verbleiben. Es gibt sie ebenfalls imprägniert mit Silber oder Antiseptika. Sie dienen dem Sekretmanagement und nehmen die Feuchtigkeit aus der Wunde auf, indem sie diese in der Beschichtung binden. Die Anwendung bei infizierten Wunden ist kontraindiziert. Beim Verbandswechsel zeigt sich das absorbierte Sekret in der Regel als durch Keimbelastung foetides Gel. Aufgrund dieses Sekrets und des Geruchs ist die Akzeptanz beim Patienten oft eingeschränkt [7].

\section{Geschlossenporige Polyurethanschäume}

Diese bieten den Vorteil, dass sie in allen Stadien der Wundheilung eingesetzt werden können. Sie bieten ein sehr gutes Sekretmanagement und halten die Wunde durch die Isolationswirkung des Schaums warm. Sie können bis zu 7 Tage auf der Wunde verbleiben. In Kombination mit einem aufgebrachten Silikonnetz haften sie sehr wenig an der Wunde. Dies verringert die Schmerzen beim Verbandswechsel. Es gibt sie mit und ohne Haftrand [8].

\section{Offenporige Schaumstoffkompressen}

Diese bestehen aus Polyurethan- oder Polyvinylalkohol-Schaum. Sie werden in der Regel insbesondere im Bereich der Dermatochirurgie als temporärer Hautersatz nach 2-zeitigen Exzisionen von Tumoren zur passageren Defektdeckung eingesetzt. Sie bieten den Vorteil einer effektiven Blutstillung sowie der Granulationsförderung über die offenporige Schaumstoffoberfläche [9].

\section{Antibakterielle Wundauflagen}

Hierbei handelt es sich in der Regel um Aktivkohlewundauflagen, die durch die große Oberfläche der Aktivkohle sowohl Gerüche als auch Keime binden. Diese gibt es kombiniert mit Silber. Insbesondere die Applikationsform des Silbers in einer speziellen Wundauflage führt dazu, dass diese als einzige Wundauflage nennenswert hohe Silberionenkonzentrationen in der Wunde erreichen lässt,

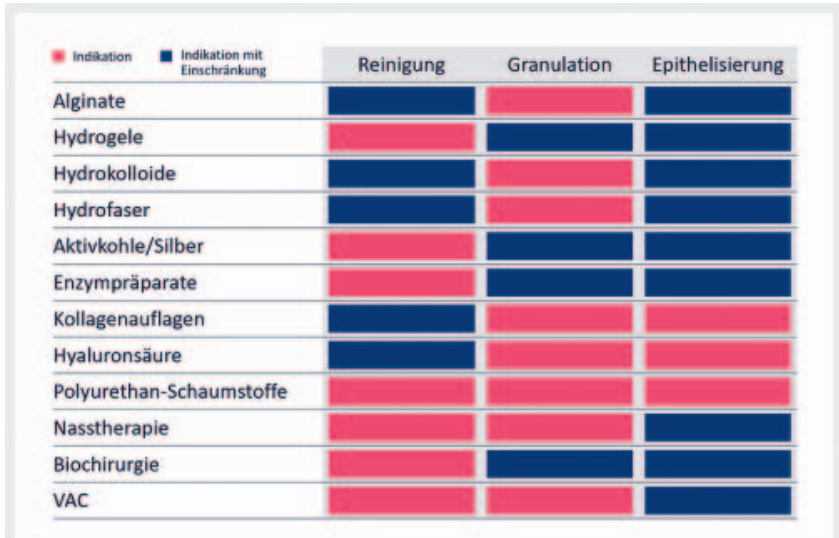

- Abb. 3 Zur Verfügung stehende Produktgruppen und Einsatzgebiete gemäß Wundheilungsphase. Insbesondere die geschlossenporigen Schaumstoffe sind für alle Phasen der Wundheilung geeignet.

die antiseptisch wirksam sind $[10,11]$. Indikationen sind hier insbesondere schlecht riechende oder infizierte Wunden.

\section{Nasstherapie}

Hierbei handelt es sich um einen Polyacrylat-Superabsorber. Dieser wird vorbefeuchtet bzw. nass auf die Wunde aufgebracht. Er erzeugt eine Spül- und Reinigungswirkung in der Wunde und hält die Wunde sehr feucht. Indikationen sind insbesondere infizierte, belegte und sehr trockene Wunden. Das Handling im ambulanten Bereich gestaltet sich jedoch für den Patienten aufgrund der kürzeren Verweildauer auf der Wunde und der damit häufigeren Verbandswechsel oft schwierig [12].

\section{Enzymatische Wundheilung}

Hier stehen mittlerweile unterschiedliche Produkte verschiedener Hersteller zur Verfügung, die über den Einsatz von Enzymen bzw. Wachstumsfaktoren die Wundheilung fördern sollen. Die Evidenz zu diesen Präparaten ist jedoch mäßig [13] ( Abb. 3).

\section{Lösungsorientierter Vorgehensansatz}

Da die Wundversorgung ein sehr emotional besetztes Thema ist und hier die einzelnen Vorlieben der Behandler im Vordergrund stehen, ist es wichtig, bei der Straffung des Wundauflagensortiments, gerade in einem größeren Klinikverbund, alle Beteiligten mitzunehmen. Hierbei spielen vor allem auch die Ziele des Einkaufs eine Rolle, die in der Regel in der Reduktion der Lieferanten und damit Erhöhung der jeweiligen Bestellmengen, die eine Preisreduktion zur Folge haben, liegen. Aber auch die einzelnen Kliniken und die Behandler vor Ort müssen in ihrem Bewusstsein bestärkt werden, dass unterschiedliche Wundbehandlungsziele mit ähnlichen Wundauflagen erreichbar sind. Damit werden sie im Prozess der Straffung des Wundauflagensortiments mitgenommen, um im Anschluss eine gute Akzeptanz dieser Entscheidung zu erreichen.

Daher wurde von uns als erstes ein Fragebogen mit den unterschiedlichen eingesetzten Produktgruppen entwickelt und an die 


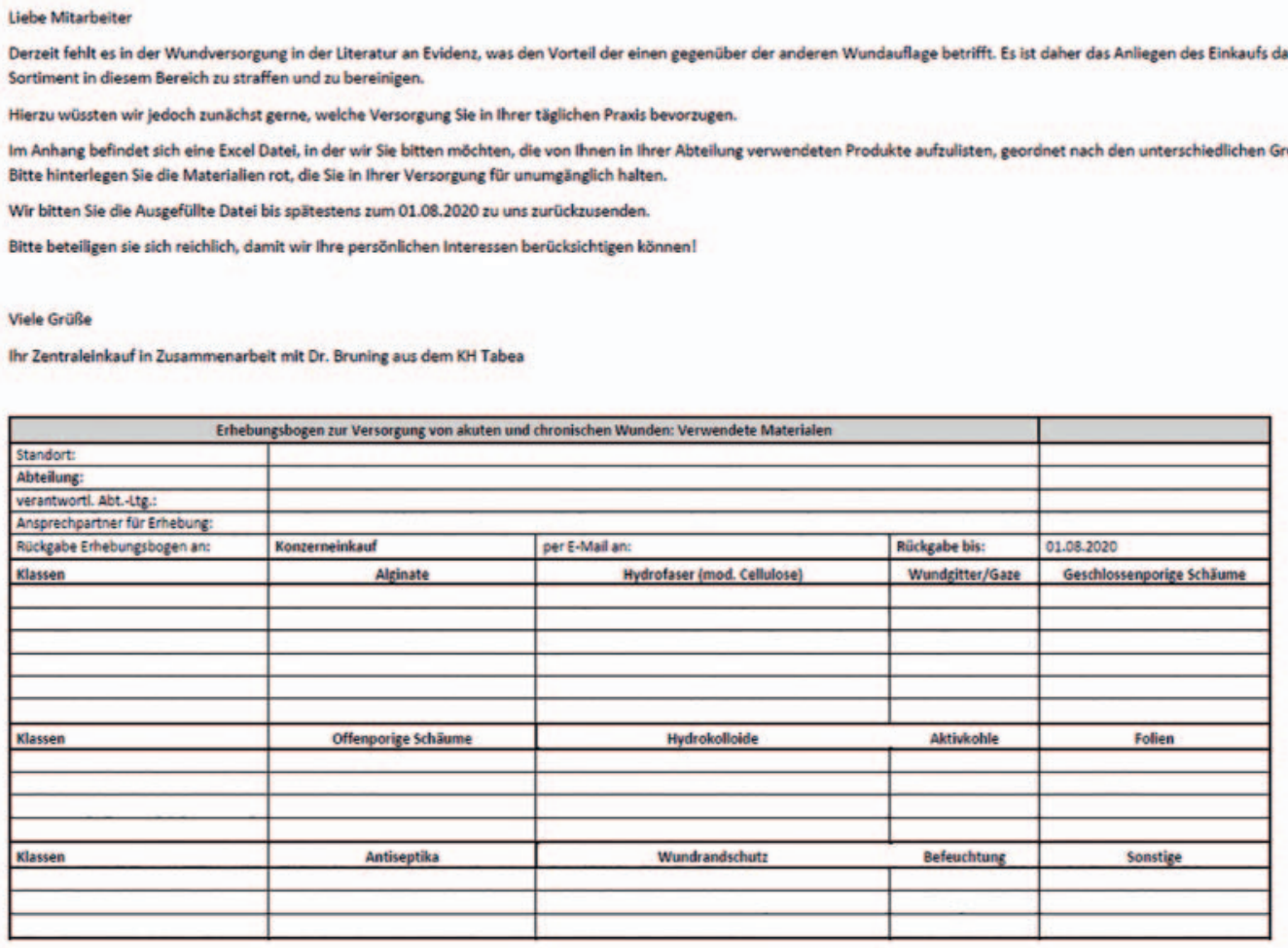

- Abb.4 Erhebungsfragebogen zur Versorgungssituation in den verschiedenen Kliniken.

einzelnen Kliniken verschickt ( $\triangleright$ Abb.4). Dadurch hatte jeder die Möglichkeit, die von ihm benutzten Produkte den entsprechenden Produktklassifikationen zuzuordnen. Dieser Prozess führt auch dazu, dass hier innerhalb der Kliniken bereits erste Überlegungen stattfinden, welche Produkte miteinander austauschbar sind. Auch die Ziele dieser Erhebung und die Gründe für den Wunsch nach Straffung des Produktsortiments wurden in dieser Phase der Befragung den entsprechenden Kliniken dargelegt. Nur 5 der 16 Kliniken haben an dieser Befragung teilgenommen und die Fragebögen zurückgeschickt. Schon hier zeigte sich die unterschiedliche Ausstattung der einzelnen Kliniken mit Produkten, die alle die gleichen Ziele in der Wundheilung haben und in der gleichen Wundheilungsphase eingesetzt werden. In Zusammenarbeit mit dem Zentraleinkauf, der sich allein aus organisatorischen und kostenpolitischen Gründen auf einen Hersteller festgelegt hat, wurde im primären Bestellsystem aus jeder Produktgruppe ein Produkt in das Sortiment aufgenommen. Im Vordergrund standen hier, aufgrund der fehlenden medizinischen Evidenz, Kosten- und organisatorische Argumente.

Primär gelistet wurde jeweils ein Produkt aus den unterschiedlichen Kategorien von einem einzigen Hersteller. Im Nachgang wurden weitere Produkte bei speziellen Indikationen ins Sortiment aufgenommen: Eine Silikongaze, welche noch bessere Antihafteigenschaften als eine normale Wundgaze aufweist, und eine Wundgaze, die in der Lage ist, aufgrund ihres besonderen Mechanismus Keime an die Wundgaze zu binden, sowie ein Aktivkohleprodukt mit Silberimprägnierung und hoher Silberfreisetzung. Durch diese Wünsche konnten auch alle nach der Umstellung

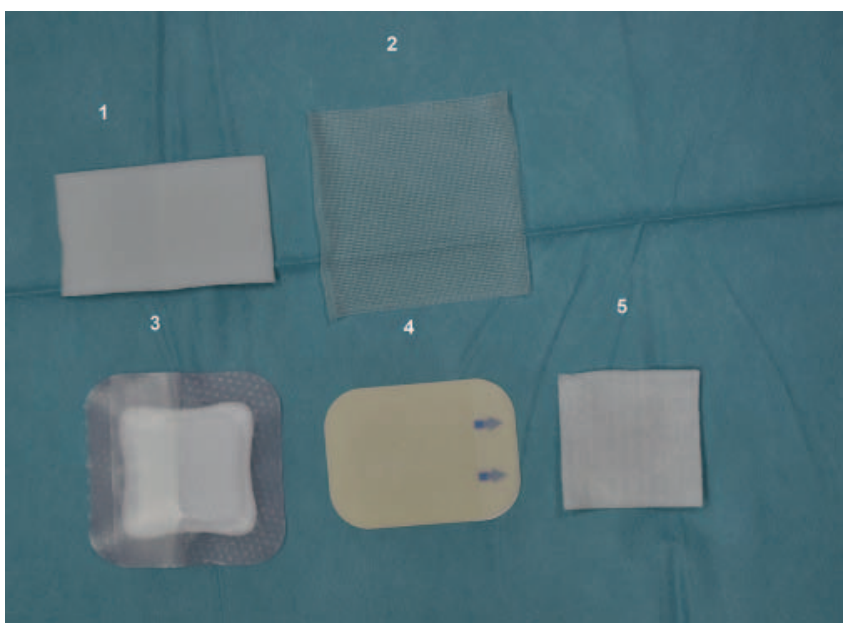

- Abb. 5 Grundlagengensortiment der Wundversorgung: 1) offenporige Schaumkompresse, 2) Wundgaze, 3) Ggeschlossenporige Schaumstoffkompresse, 4) Hydrokolloid und 5) Hydrofaser.

geäußerten Kritikpunkte aus den einzelnen Kliniken befriedigt werden. Ergänzt wird dieses Produktportfolio durch Antiseptika aus dem Polyhexanid- Bereich sowie ein Acrylat-Terpolymer zum Wundrandschutz ( $\triangleright$ Abb. 5, 6). 


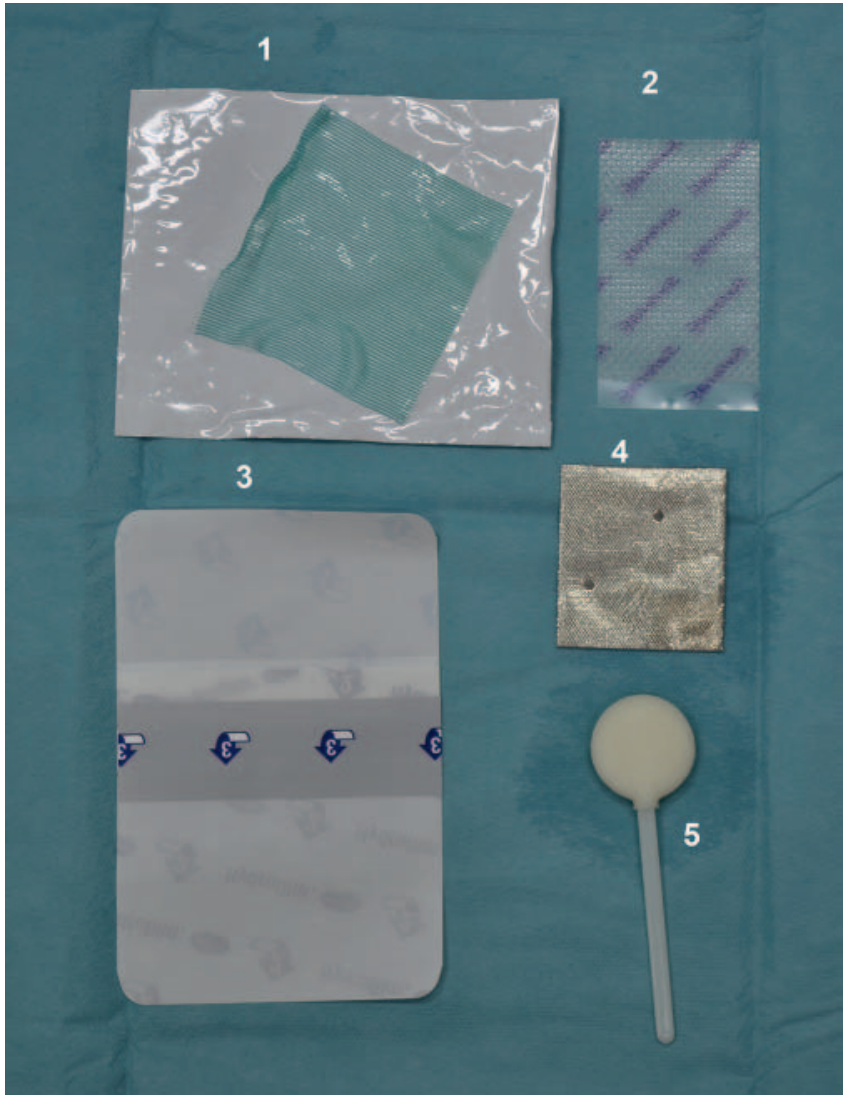

- Abb. 6 Ergänzungsbedarf: 1) Wundgaze mit keimabsorbierender Beschichtung, 2) Silikonwundgitter, 3) Folienverband, 4) Aktivkohleprodukt mit hoher Silberfreisetzung und 5) Acrylat- TerpolymerLösung zum Wundrandschutz mit Applikationslolli.

\section{Fazit und Zusammenfassung}

Die große Akzeptanz über alle Kliniken hinweg zeigt, dass die Straffung des Wundauflagensortiments auch innerhalb der Kliniken auf große Befürwortung stößt. Durch den Umstrukturierungsprozess ist die Sensibilität der Mitarbeiter im Umgang mit Wundauflagen geschärft worden und es wurde ein besseres Verständnis für die bestehende Problematik geschaffen. Es finden seltener unbegründete Therapiewechsel statt und die Einarbeitung der Mitarbeiter ist deutlich erleichtert. Aufgrund der fehlenden Evidenz für die meisten Wundauflagen ist es berechtigt, bei der Entscheidung für einzelne Komponenten im eigenen Sortiment Prozessoptimierungs- und Kostenargumente in den Vordergrund zu stellen, ohne dass die Qualität der Patientenversorgung leidet.
Interessenkonflikt

Die Autorinnen/Autoren geben an, dass kein Interessenkonflikt besteht.

\section{Literatur}

[1] S-leitlinie K. Deutsche Gesellschaft für Wundheilung und Wundbehandlung e.V. https://www.dga-gefaessmedizin.de/uploads/media/ S3_LL_Lokaltherapie_chronischer_Wunden_2012-06.pdf

[2] Cutting K, McGuire J. In vitro and clinical experience of Cutimed Sorbact: the evidence base. J Wound Care 2015; 24: S6-S30. doi:10.12968/ jowc.2015.24.Sup5a.S6

[3] Beele $\mathrm{H}$, Meuleneire F, Nahuys $\mathrm{M}$ et al. A prospective randomised open label study to evaluate the potential of a new silver alginate/carboxymethylcellulose antimicrobial wound dressing to promote wound healing. Int Wound J 2010; 7: 262-270. doi:10.1111/j.1742481X.2010.00669.x

[4] Aderibigbe BA, Buyana B. Alginate in wound dressings. Pharmaceutics 2018; 10: doi:10.3390/pharmaceutics10020042

[5] Barnea Y, Weiss J, Gur E. A review of the applications of the hydrofiber dressing with silver (Aquacel Ag) in wound care. Ther Clin Risk Manag 2010; 6: 21-27. doi:10.2147/tcrm.s3462

[6] Francesko A, Petkova P, Tzanov T. Hydrogel Dressings for Advanced Wound Management. Curr Med Chem 2018; 25: 5782-5797. doi:10.2174/0929867324666170920161246

[7] Jurić Vukelić D, Jurić J. Hydrocolloid Dressing Application in the Treatment of Chronic Wounds and Relation to Quality of Life. Acta Clin Croat 2017; 56: 544-549. doi:10.20471/acc.2017.56.03.22

[8] Franks PJ, Moody M, Moffatt C] et al. Randomized trial of two foam dressings in the management of chronic venous ulceration. Wound repair Regen Off Publ Wound Heal Soc [and] Eur Tissue Repair Soc 2007; 15: 197-202. doi:10.1111/j.1524-475X.2007.00205.x

[9] Stone HA, Edelman RD, McGarry J]. Epigard: a synthetic skin substitute with application to podiatric wound management. J foot ankle Surg Off Publ Am Coll Foot Ankle Surg 1993; 32: 232-238

[10] Gago M, Garcia F, Gaztelu V et al. A Comparison of Three Silver-containing Dressings in the Treatment of Infected, Chronic Wounds. Wounds a Compend Clin Res Pract 2008; 20: 273-278

[11] Nešporová K, Pavlík V, Šafránková B et al. Effects of wound dressings containing silver on skin and immune cells. Sci Rep 2020; 10: 1-14. doi:10.1038/s41598-020-72249-3

[12] Ousey K, Rogers AA, Rippon MG. Hydro-responsive wound dressings simplify T.I.M.E. wound management framework. Br J Community Nurs 2016; 21 (Suppl. 12): S39-S49. doi:10.12968/bjcn.2016.21.Sup12.S39. PMID: 27922787

[13] Shoham $Y$, Krieger $Y$, Tamir E et al. Bromelain-based enzymatic debridement of chronic wounds: A preliminary report. Int Wound J 2018; 15 : 769-775. doi:10.1111/iwj.12925 